\title{
The negative bone effects of the disease and of chronic corticosteroid treatment in premenopausal women affected by rheumatoid arthritis
}

\author{
A. Fassio, L. Idolazzi, M.A. Jaber, C. Dartizio, O. Viapiana, M. Rossini, D. Gatti \\ Rheumatology Connected Operative Unit, University of Verona, Verona, Italy
}

\begin{abstract}
SUMMARY
Osteoporosis is a well-known extra-articular complication in rheumatoid arthritis (RA). The chronic corticosteroid treatment, the functional impairment associated with RA and the disease itself appear to be the most relevant determinants. Most of the previous studies involved postmenopausal women, in whom the estrogenic deficiency might amplify the negative effect towards bone of both RA and corticosteroid therapy. We decided to evaluate bone health in a cohort of premenopausal RA patients. The study population includes 47 premenopausal women attending our outpatient clinic for RA and twice as many healthy age-matched control women selected from the hospital personnel. The bone density at the spine and femoral neck were significantly lower in patients with RA as compared with controls. When spine bone mineral density (BMD) values were adjusted for the cumulative glucocorticoid (GC) dose alone and for the cumulative GC dose plus body mass index (BMI) the mean differences between two groups decreased but they remained statistically significant. We found no difference when the spine BMD was adjusted for cumulative GC dose, BMI and health assessment questionnaire. The difference in femoral neck BMD remained statistically significant also after all the same adjustments.

In conclusion, our study shows that a BMD deficiency is frequent also in premenopausal women affected by RA, especially at femoral site and that the main determinants of this bone loss are not only the disease-related weight loss, corticosteroid therapy and functional impairment, but also the systemic effects of the disease itself.
\end{abstract}

Key words: Rheumatoid arthritis; glucocorticoid therapy; premenopausal women.

Reumatismo, 2016; 68 (2): 65-71

\section{INTRODUCTION}

steoporosis is a well-known extraarticular complication in rheumatoid arthritis (RA) $(1,2)$ and an increased risk of fractures has been clearly documented in patients with RA (1-7). The pathogenesis of the decreased bone mineral density (BMD) values at the hip, spine and total body reported in a number of studies $(1,4$, $8-12)$ is multifactorial $(2,13-15)$. Chronic glucocorticoid (GC) treatment, together with the functional impairment associated with RA appear to be the most relevant determinants $(4,6,14,16)$, even though the disease itself may play a relevant role too $(17,18)$. Recently, RA has been taken into account as an independent risk factor in the assessment of fracture risk $(19,20)$.

In all previous studies, the study popula- tion was almost invariably made of postmenopausal women where the estrogenic deficiency might amplify the negative effect of RA and corticosteroid therapy on skeletal health.

Indeed, all the major scientific societies recommend a specific treatment in postmenopausal women receiving GC therapy particularly when they are affected by RA (21). The aim of our study was to compare bone mass and mineral metabolism in a cohort of premenopausal women and in a group of age-matched healthy women.

\section{MATERIALS AND METHODS}

The study population includes 47 premenopausal women (last menses less than 40 days before the index visit) attending to our outpatient clinic for RA and twice as $\overline{\text { Corresponding author }}$ Angelo Fassio

Rheumatology Connected Operative Unit, University of Verona

Piazzale Aristide Stefani, 1

37126 Verona, Italy

E-mail: angelo.fassio@yahoo.it 
many healthy age-matched control women selected form the hospital personnel. All patients fulfilled the revised 1987 American College of Rheumatology (22).

We evaluated: mobility activities of daily living score (23), Steinbrocker functional state (1 to 4) (24), erythrocyte sedimentation rate, rheumatoid factor, anti-cyclic citrullinated peptide and routine biochemistry. Additional information included: disease onset, extra-articular manifestations, smoking and alcohol intake habits, treatments specific for RA and osteoporosis.

Vitamin D supplements, taken during the previous year, were carefully evaluated and expressed as mean daily dose even when vitamin $\mathrm{D}$ was taken intermittently. Exposure to sunlight from March to September was quantified as $<20 \mathrm{~min}, 20$ to $40 \mathrm{~min}$ and $>40$ min daily. Daily intake of calcium was assessed by a simplified validated questionnaire (25). In all subjects body weight and height (Harpender stadiometer) were assessed and the body mass index $\left(\mathrm{BMI}=\mathrm{kg} / \mathrm{m}^{2}\right)$ calculated.

Individual aliquots of serum samples were collected from September to December 2008 from each woman and kept at $-70^{\circ} \mathrm{C}$ for the measurements of serum intact parathyroid hormone and 25 hydroxy-vitamin D [25(OH)D] by commercial ELISA kits (IDS Co., Bolden, UK) with inter-assay coefficient of variations ranging from 5 to $15 \%$.

A Hologic Delphi instrument executed the dual-energy X-ray absorptiometry (DXA) evaluation of the BMD at the lumbar spine and femoral neck. The in vivo coefficient of variation was $0.7 \%$ at the spine and $1.2 \%$ at the hip. The BMD values were expressed both as absolute values and as Tscores. T-score is the number of standard deviations below the average for a young adult at peak bone density. The World Health Organization has defined the following categories based on bone density in white women (26):

1) normal bone: T-score better than -1 ;

2) osteopenia: T-score between -1 and -2.5 ;

3) osteoporosis: T-score less than -2.5 ;

4) established (severe) osteoporosis includes the presence of a non-traumatic fracture.

The differences between control women and patients for continuous values were tested by unpaired t-test. ANCOVA was used to test significance for BMD values adjusted for all potential interfering variable. Statistical analysis was realized by SPSS version 11 (SPSS Inc., Chicago, IL, USA).

\section{RESULTS}

The baseline main features of the two groups are reported in Table I.

We found no difference in biochemical markers of bone metabolism. Levels of 25(OH)D lower than $20 \mathrm{ng} / \mathrm{mL}$ (27) was found in $38.3 \%(18 / 47)$ and $38.1 \%$ (37/94)

Table I - Main features of the two groups.

\begin{tabular}{|l|l|l|l|l|}
\hline \multicolumn{2}{|c}{} & No. & Mean \pm SD & P \\
\hline Age (years) & Controls & 94 & $41.9 \pm 5.9$ & NS \\
& RA & 47 & $42.2 \pm 5.7$ & \\
\hline BMI (Kg/m2) & Controls & 94 & $24.1 \pm 4.4$ & $<0.05$ \\
& RA & 47 & $22.6 \pm 3.2$ & \\
\hline 25 OH Vit D (ng/mL) & Controls & 94 & $23.1 \pm 9.2$ & NS \\
& RA & 47 & $24.1 \pm 11.0$ & \\
\hline CTX (ng/mL) & Controls & 94 & $0.29 \pm 0.14$ & NS \\
& RA & 47 & $0.31 \pm 0.14$ & \\
\hline PTH (pg/mL) & Controls & 94 & $26.8 \pm 10.9$ & NS \\
& RA & 47 & $23.7 \pm 11.6$ & \\
\hline Smoking subjects & Controls & 94 & $19 / 94=20.2 \%$ & NS \\
& RA & 47 & $9 / 47=19.1 \%$ & \\
\hline
\end{tabular}

$\mathrm{SD}$, standard deviation; RA, rheumatoid arthritis; BMI, body mass index; CTX, bone turnover marker; PTH, serum intact parathyroid hormone; NS, not significant. 
Table II - Disease related variables in the 47 patients affected by rheumatoid arthritis.

\begin{tabular}{|l|l|l|l|}
\hline & Mean \pm SD & Minimum & Maximum \\
\hline Disease duration (years) & $9 \pm 7$ & 2 & 25 \\
\hline Steinbrocker's classification & $1.6 \pm 0.7$ & 1 & 4 \\
\hline HAQ & $0.6 \pm 0.6$ & 0.00 & 2.62 \\
\hline Number of swollen joints & $2.4 \pm 3.6$ & 0 & 20 \\
\hline ESR $(\mathrm{mm} / \mathrm{h})$ & $28.1 \pm 22.4$ & 4 & 97 \\
\hline Prednisone equivalent daily dose $(\mathrm{mg})^{*}$ & $5.3 \pm 1.7$ & 2 & 8 \\
\hline Months of corticosteroid therapy $^{*}$ & $28.1 \pm 33.7$ & 6 & 127 \\
\hline Cumulative dose (mg prednisone equivalent) $^{*}$ & $776 \pm 917$ & 150 & 4500 \\
\hline
\end{tabular}

$\mathrm{SD}$, standard deviation; $\mathrm{HAQ}$, health assessment questionnaire; ESR, erythrocyte sedimentation rate. *Relative only to the 37 patients on corticosteroid therapy.

of the patients and controls, respectively (thus confirming what was already found in different rheumatic diseases (28) (data not shown). Mean BMI was statistically lower in RA patients $(\mathrm{P}<0.05)$.

The main disease related findings of the 47 patients affected by RA are listed in Table II.

Steroid treatment was underway in 37 of 47 patients while 10 subjects never received treatment with corticosteroids. The features of steroid therapy relative only to the 37 patients on corticosteroid therapy were (Table II): prednisone equivalent mean daily dose (mg) $5.3 \pm 1.7 \mathrm{mg}$, mean duration of corticosteroid therapy $28.1 \pm 33.7$ months and mean cumulative dose $776 \pm 917 \mathrm{mg}$ (prednisone equivalent).
The bone densities at spine and femoral neck were significantly lower in patients with RA as compared with controls.

The proportion of patients with osteopenia and osteoporosis at the spine were 32 and $11 \%$ in patients vs 17 and $0 \%$ in controls. The corresponding proportion of patients with osteopenia and osteoporosis at the femoral neck were 26 and $13 \%$ in patients vs 4 and $0 \%$ in controls respectively.

As compared with control group, the relative hazard to be osteopenic or osteoporotic was 3.61 [95\% confidence interval $(\mathrm{CI})=1.64$ to 7.95$]$ and $13.97(95 \% \mathrm{CI}=4.37$ to 44.61 ) at spine and femoral neck respectively.

The BMD differences were 7.9 and $16.9 \%$ at the spine and the femoral neck respec-

Table III - Comparison of spine and femoral bone mineral density data expressed as absolute values in rheumatoid arthritis and in control groups with and without adjustment for cumulative corticosteroid dose, body mass index and health assessment questionnaire.

\begin{tabular}{|l|l|l|l|l|}
\hline Spine & RA & Controls & Diff\% & P \\
\hline Not adjusted & $965.8 \pm 131.1$ & $1048.8 \pm 118.8$ & -7.9 & $<0.001$ \\
\hline Adj. for CORT & $981.5 \pm 134.4$ & $1041.0 \pm 130.0$ & -5.7 & $<0.02$ \\
\hline Adj. for CORT+BMI & $983.9 \pm 132.9$ & $1040.0 \pm 128.9$ & -5.4 & $<0.03$ \\
\hline Adj. for CORT+BMI+HAQ & $1029.0 \pm 153.3$ & $1020.0 \pm 136.7$ & 0.0 & NS \\
\hline \multicolumn{5}{|l|}{} \\
\hline Femoral neck & RA & Controls & Diff\% & P \\
\hline Not adjusted & $760.3 \pm 175.7$ & $915.0 \pm 112.1$ & -16.9 & $<0.001$ \\
\hline Adj. for CORT & $788.6 \pm 145.4$ & $903.5 \pm 136.7$ & -12.7 & $<0.001$ \\
\hline Adj. for CORT+BMI & $792.7 \pm 137.5$ & $901.8 \pm 128.9$ & -12.0 & $<0.001$ \\
\hline Adj. for CORT+BMI+HAQ & $814.9 \pm 163.9$ & $892.9 \pm 140.6$ & -8.7 & $<0.03$ \\
\hline
\end{tabular}

RA, reumatoid arthritis; CORT, corticosteroid; BMI, body mass index; HAQ, health assessment questionnaire; NS, not significant. 


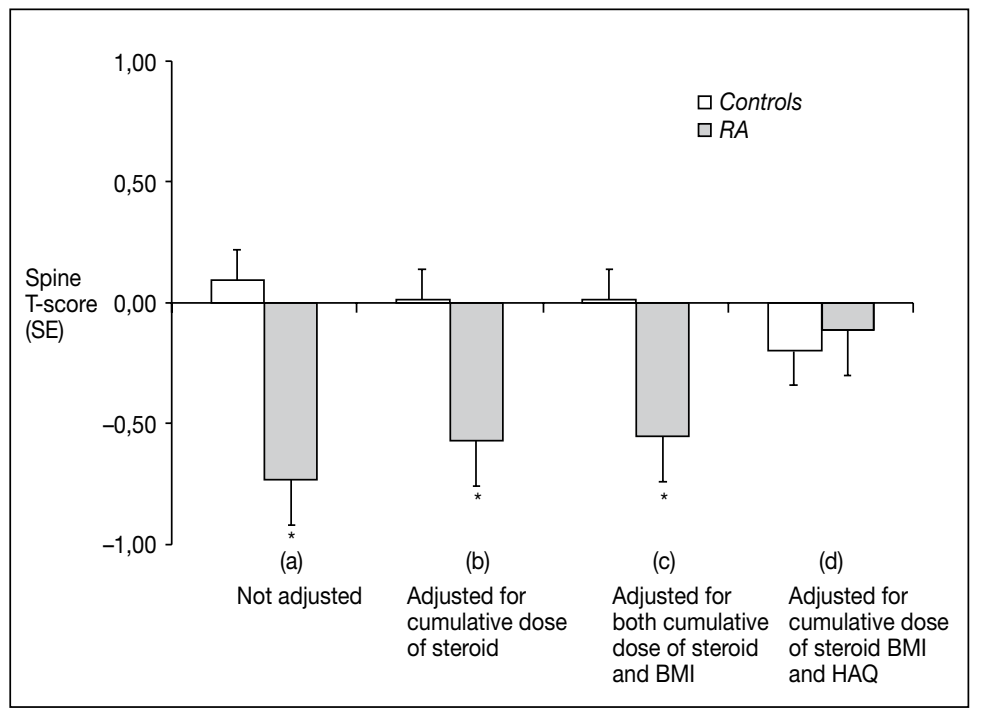

Figure 1 - Mean spine T-score values $( \pm S E)$ both un-adjusted and adjusted (see figure for adjusting variables) in patients with reumatoid arthritis and controls. ${ }^{*} \mathrm{P}<0.05$ vs control subjects.

tively (Table III). When spine BMD values were adjusted for the cumulative GC dose alone and for the cumulative GC dose plus BMI the mean differences decreased to 5.7 and to $5.4 \%$ respectively but they remained statistically significant (Table III; Figure 1). No difference was found when spine BMD was adjusted for

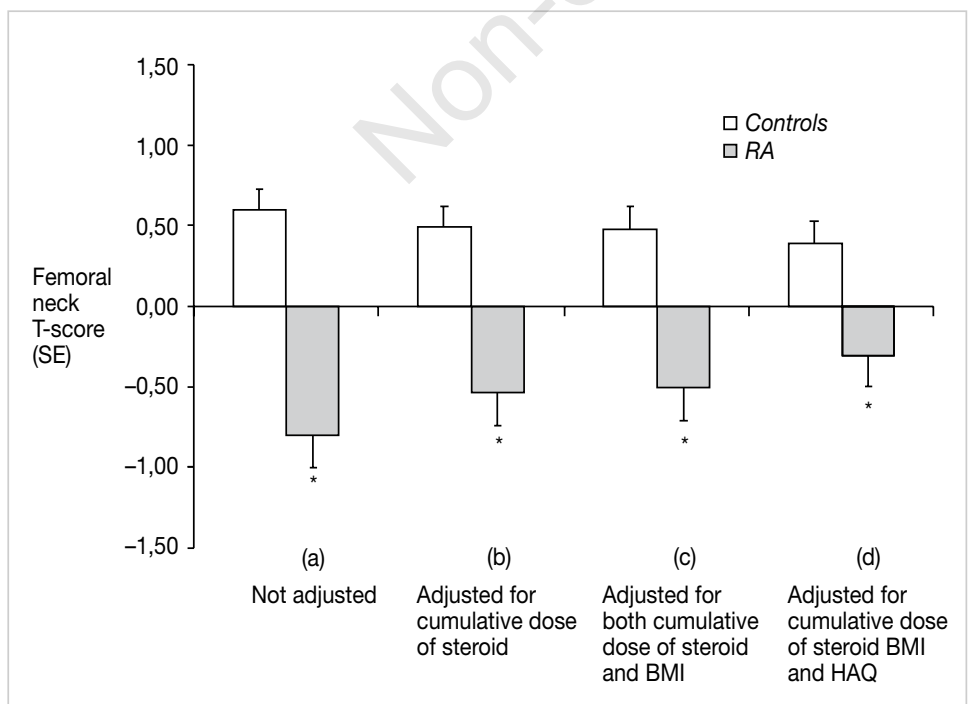

Figure 2 - Mean femoral neck T-score values $( \pm S E)$ both un-adjusted and adjusted (see figure for adjusting variables) in patients with reumatoid arthritis and controls. ${ }^{*} \mathrm{P}<0.05$ vs control subjects. cumulative GC dose, BMI and health assessment questionnaire (HAQ) (Table III; Figure 1).

The difference in femoral neck BMD remained statistically significant also after all adjustment (cumulative GC dose alone, cumulative GC dose plus BMI, and cumulative GC dose plus BMI plus HAQ) although their extents gradually decreased from 16.9 to $8.7 \%$ (Table III; Figure 2).

\section{DISCUSSION AND CONCLUSIONS}

In this study we have shown that that in premenopausal women with RA both spine and hip BMD values are significantly lower than in age-matched controls and that this difference is maintained at the hip when the BMD values are adjusted for GC therapy and the indices of disease activity. This suggests that the disease itself is responsible of significant bone loss, particularly at skeletal sites made of predominantly cortical bone.

An association between RA and low BMD values has been reported in a number of studies but a proper controlled study has been carried out only by Gough and colleagues (2). In this study Gough and his colleagues performed a DXA prospective evaluation (at 12 and 24 months) in a group of patients with RA (disease duration less than 2 years) and controls showing that RA patients tended to lose bone significantly faster than controls at all sites.

All these studies, however, included a large proportion of post-menopausal women, in whom estrogen deficiency might amplify the negative effect of RA and corticosteroid therapy on bone metabolism.

Hämäläinen and colleagues (29) studied the changes in BMD in 74 premenopausal women with RA during a two-year followup with DXA-BMD measurements in the lumbar spine and left femoral neck. The patients with RA treated with prednisone had lower BMD values than those without this treatment present at the start of the follow-up. Mean BMD decreased significantly in both lumbar spine and femoral 
neck only in the RA group treated with corticosteroids.

Tourinho and colleagues (30) studied 78 premenopausal patients with RA and 39 controls and showed a significantly lower lumbar spine BMD in the patients. Lumbar spine osteopenia correlated with no physical activity at work status, low body weight, and duration of GC therapy.

In our study too, an important determinant of low BMD values was BMI, which was significantly lower in patients than in controls $\left(22.6 \pm 3.2 v s 24.1 \pm 4.4 \mathrm{~kg} / \mathrm{m}^{2}\right)$. Body weight and BMI are among the most relevant determinants of BMD values (31) and our finding should be evaluated in the context of the strong relationship between BMI with disease activity $(32,33)$.

We found no differences in biochemical markers. The same high prevalence of vitamin D deficiency $(25(\mathrm{OH}) \mathrm{D}$ levels lower than $20 \mathrm{ng} / \mathrm{mL}$ ) was found in 2 groups: $38.3 \%$ (18/47) in RA patients vs $38.1 \%$ (37/94) in controls.

No differences in bone turnover markers were shown and these are important data, given that a large proportion (37/47: about $80 \%$ ) of RA patients were in steroid treatment.

The crude BMD reductions in RA patients $v s$ controls were statistically significant (by 8.3 and $17.1 \%$ at spine and femoral neck respectively) (Table III; Figures 1 and 2).

The RA patients were slimmer as likely effect of chronic inflammation and disease and we decided to adjust BMD values by BMI (ANCOVA) (Table III; Figures 1 and $2)$. The differences remained significant and only slightly decreased after this adjustment and this fact shows that the BMD reduction is due only in small part to disease related weight loss.

The corticosteroid therapy is known as a very important cause of bone mass reduction $(34,35)$. Of our 47 patients, 37 were on GC therapy whereas only 10 patients never assumed steroids. BMD values were then adjusted (ANCOVA) for both the cumulative GC dose and BMI (Table III; Figures 1 and 2). The differences with the control group decreased to
5.4\% $(\mathrm{P}<0.03)$ and $12.1 \%(\mathrm{P}<0.001)$ at the spine and hip, but remained statistically significant.

This fact also proves that in premenopausal women the BMD defect in RA is not due at all to corticosteroid therapy. Finally, we wanted see the weight of disability on RA osteoporosis. The BMD data were also adjusted for HAQ-DI as well as for BMI and cumulative GC dose (Table III; Figures 1 and 2). The adjusted difference of BMD values $v s$ control subjects remained statistically significant $(\mathrm{P}<0.03)$ even if only at the femoral neck ( $8.7 \%$ difference).

Thus, it seems that the cumulative dose of GC, the effect of the disease on weight and the disability are together able to justify the densitometric deficit at the lumbar spine (cancellous bone). On the contrary, at the femur (cortical bone), the correction for those parameters (however relevant) is able to decrease but not to eliminate the densitometric gap when compared to controls. A possible further cause might be, in our opinion, the disease itself, which may be able to cause negative effect on the cortical bone not only locally, but also in a systemic manner (17).

The main results of our study show that a BMD defect is also a frequent feature in premenopausal women with RA and that this defect is more evident at femoral site. This fact confirms a preferential effect of RA on cortical bone (18).

Indeed, the role of the cumulative dose of GC, BMI and HAQ is demonstrated by the observation that, after the correction of data concerning BMD for these parameters, the differences decrease (femur) or nullify (lumbar spine). However, the persistence of a difference at the femur supports the hypothesis that also the disease itself can justify the BMD deficit at the densitometry.

Conflict of interest: the authors declare no potential conflict of interest.

Acknowledgments: the authors would like to thank the laboratory teams, especially Caterina Fraccarollo for performing the biochemical analyses. 


\section{REFERENCES}

1. Spector TD, Hall GM, McCloskey EV, Kanis JA. Risk of vertebral fracture in women with rheumatoid arthritis. BMJ. 1993; 306: 558.

2. Gough AK, Lilley J, Eyre S, et al. Generalised bone loss in patients with early rheumatoid arthritis. Lancet Lond Engl. 1994; 344: 23-7.

3. Hooyman JR, Melton LJ, Nelson AM, et al. Fractures after rheumatoid arthritis. A population-based study. Arthritis Rheum. 1984; 27 : 1353-61.

4. Peel NF, Moore DJ, Barrington NA, et al. Risk of vertebral fracture and relationship to bone mineral density in steroid treated rheumatoid arthritis. Ann Rheum Dis. 1995; 54: 801-6.

5. Michel BA, Bloch DA, Fries JF. Predictors of fractures in early rheumatoid arthritis. J Rheumatol. 1991; 18: 804-8.

6. Cooper C, Coupland C, Mitchell M. Rheumatoid arthritis, corticosteroid therapy and hip fracture. Ann Rheum Dis. 1995; 54: 49-52.

7. Başkan BM, Sivas F, Alemdaroğlu E, et al. Association of bone mineral density and vertebral deformity in patients with rheumatoid arthritis. Rheumatol Int. 2007; 27: 579-84.

8. Martin WF, Cerundolo LH, Pikosky MA, et al. Effects of dietary protein intake on indexes of hydration. J Am Diet Assoc. 2006; 106: 587-9.

9. Sambrook PN, Eisman JA, Champion GD, et al. Determinants of axial bone loss in rheumatoid arthritis. Arthritis Rheum. 1987; 30: 721-8.

10. Hall GM, Spector TD, Griffin AJ, et al. The effect of rheumatoid arthritis and steroid therapy on bone density in postmenopausal women. Arthritis Rheum. 1993; 36: 1510-6.

11. Kröger H, Honkanen R, Saarikoski S, Alhava E. Decreased axial bone mineral density in perimenopausal women with rheumatoid arthritis-a population based study. Ann Rheum Dis. 1994; 53: 18-23.

12. Innala L, Sjöberg C, Möller B, et al. Co-morbidity in patients with early rheumatoid arthritis - inflammation matters. Arthritis Res Ther. 2016; 18: 33.

13. Solomon DH, Finkelstein JS, Shadick N, et al. The relationship between focal erosions and generalized osteoporosis in postmenopausal women with rheumatoid arthritis. Arthritis Rheum. 2009; 60: 1624-31.

14. Coulson KA, Reed G, Gilliam BE, et al. Factors influencing fracture risk, $\mathrm{T}$ score, and management of osteoporosis in patients with rheumatoid arthritis in the Consortium of Rheumatology Researchers of North America (CORRONA) registry. J Clin Rheumatol Pract Rep Rheum Musculoskelet Dis. 2009; 15: 155-60.

15. Sambrook PN, Eisman JA, Yeates MG, et al.
Osteoporosis in rheumatoid arthritis: safety of low dose corticosteroids. Ann Rheum Dis. 1986; 45: 950-3.

16. Lems WF, Jahangier ZN, Jacobs JW, Bijlsma JW. Vertebral fractures in patients with rheumatoid arthritis treated with corticosteroids. Clin Exp Rheumatol. 1995; 13: 293-7.

17. Rossini M, Viapiana O, Adami S, et al. In patients with rheumatoid arthritis, Dickkopf-1 serum levels are correlated with parathyroid hormone, bone erosions and bone mineral density. Clin Exp Rheumatol. 2015; 33: 77-83.

18. Sinigaglia L, Nervetti A, Mela Q, et al. A multicenter cross sectional study on bone mineral density in rheumatoid arthritis. Italian Study Group on Bone Mass in Rheumatoid Arthritis. J Rheumatol. 2000; 27: 2582-9.

19. Hippisley-Cox J, Coupland C. Predicting risk of osteoporotic fracture in men and women in England and Wales: prospective derivation and validation of QFractureScores. BMJ. 2009; 339: b4229.

20. Kanis JA, Johnell O, Oden A, et al. FRAX and the assessment of fracture probability in men and women from the UK. Osteoporos Int J Establ Result Coop Eur Found Osteoporos Natl Osteoporos Found USA. 2008; 19: 385-97.

21. Rossini M, Orsolini G, Viapiana O, et al. Bisphosphonates in the treatment of glucocorticoid-induced osteoporosis: pros. Endocrine. 2015; 49: 620-7.

22. Arnett FC, Edworthy SM, Bloch DA, et al. The American Rheumatism Association 1987 revised criteria for the classification of rheumatoid arthritis. Arthritis Rheum. 1988; 31: 315-24.

23. Fries JF, Spitz P, Kraines RG, Holman HR. Measurement of patient outcome in arthritis. Arthritis Rheum. 1980; 23: 137-45.

24. Lawton MP, Brody EM. Assessment of older people: self-maintaining and instrumental activities of daily living. The Gerontologist. 1969; 9: 179-86.

25. Steinbrocker O, Traeger $\mathrm{CH}$, Batterman RC. Therapeutic criteria in rheumatoid arthritis. J Am Med Assoc. 1949; 140: 659-62.

26. Adami S, Viapiana O, Gatti D, et al. Relationship between serum parathyroid hormone, vitamin D sufficiency, age, and calcium intake. Bone. 2008; 42: 267-70.

27. [No authors listed]. Assessment of fracture risk and its application to screening for postmenopausal osteoporosis. Report of a WHO Study Group. World Health Organ Tech Rep Ser. 1994; 843: 1-129.

28. Adami S, Romagnoli E, Carnevale V, et al. [Guidelines on prevention and treatment of vitamin D deficiency. Italian Society for Osteoporosis, Mineral Metabolism and Bone Diseases (SIOMMMS)]. Reumatismo. 2011; 63: 129-47. [Article in Italian]. 
29. Rossini M, Gatti D, Viapiana O, et al. Vitamin D and rheumatic diseases. Reumatismo. 2014; 66: $153-70$.

30. Martin JC, Munro R, Campbell MK, Reid DM. Effects of disease and corticosteroids on appendicular bone mass in postmenopausal women with rheumatoid arthritis: comparison with axial measurements. Br J Rheumatol. 1997; 36: 43-9.

31. Hämäläinen $H$, Kaarela $K$, Kröger $H$, et al. Changes in bone mineral density in premenopausal women with rheumatoid arthritis during a two-year follow-up. Jt Bone Spine Rev Rhum. 2007; 74: 482-7.

32. Tourinho TF, Stein A, Castro JAS, Brenol JCT. Rheumatoid arthritis: evidence for bone loss in premenopausal women. J Rheumatol. 2005; 32: 1020-5.

33. De Laet C, Kanis JA, Odén A, et al. Body mass index as a predictor of fracture risk: a meta-analysis. Osteoporos Int J Establ Result Coop Eur Found Osteoporos Natl Osteoporos Found USA. 2005; 16: 1330-8.

34. Book C, Karlsson MK, Akesson K, Jacobsson LTH. Early rheumatoid arthritis and body composition. Rheumatol Oxf Engl. 2009; 48: 1128-32.

35. Giles JT, Bartlett SJ, Andersen RE, et al. Association of body composition with disability in rheumatoid arthritis: impact of appendicular fat and lean tissue mass. Arthritis Rheum. 2008; 59: 1407-15. 